\title{
Multiple Endotracheal Metastases of Lung Cancer after Bronchoscopic Intervention
}

\author{
Kazuki Hayasaka ${ }^{1}$, Satoshi Shiono ${ }^{1}$ and Naoki Yanagawa ${ }^{2}$
}

\begin{abstract}
:
A 65-year-old man presented with obstructive pneumonia due to squamous cell carcinoma of the lung, which invaded the right main bronchus. Argon plasma coagulation was performed, which reduced his symptoms. Right pneumonectomy was performed after preoperative chemotherapy; the pathological diagnosis was T3N1M0 Stage IIIA. Fifteen months after surgery, he presented with bloody sputum. Bronchoscopy showed multiple endotracheal tumors, and a pathological examination revealed squamous cell carcinoma; the same histology as the primary lung cancer. We hypothesized that direct implantation during bronchoscopic therapy or hematogenous or lymphatic spread might have led to metastasis. A careful follow-up is required after bronchoscopic therapy.
\end{abstract}

Key words: lung cancer, trachea, metastasis, bronchoscopic intervention

(Intern Med 57: 845-847, 2018)

(DOI: 10.2169/internalmedicine.9542-17)

\section{Introduction}

Endotracheal metastasis of lung cancer is very rare. Until now, only a small number of cases have been reported (1-5), and the mechanisms of metastasis remain unclear. To the best of our knowledge, no reports have described multiple endotracheal metastases of lung cancer after bronchoscopic treatment followed by surgery. We herein present an unusual case of multiple endotracheal metastases of lung cancer after bronchoscopic treatment followed by surgery.

\section{Case Report}

A 65-year-old man presented with dyspnea and fever. Chest computed tomography (CT) revealed a right hilar lung tumor and right hilar lymph node swelling that invaded the right main bronchus and obstructed the right upper lobe bronchus (Fig. 1A). Bronchoscopy revealed that the tumor completely obstructed the right upper bronchus and invaded the right main bronchus (Fig. 1B). Since this patient suffered from severe dyspnea, argon plasma coagulation (APC) was performed to relieve his symptoms. During the procedure, the tumor was coagulated by APC and removed using a forceps. After the procedure, the severity of the patient's symptoms was reduced. The pathological diagnosis was squamous cell carcinoma of the lung (clinical stage: T3N1M0, stage IIIA). To avoid pneumonectomy, preoperative chemotherapy with two courses of gemcitabine/cisplatin was administered. However, the tumor size did not decrease, so right pneumonectomy and systemic lymph node dissection were performed. The pathological diagnosis was moderately differentiated squamous cell carcinoma of the lung with direct invasion of the right main bronchus and metastasis to the right hilar lymph node (pathological stage: pT3N1M0, stage IIIA). After surgery, the patient was doing well and did not receive any adjuvant chemotherapy or radiotherapy based on the consideration of potential critical adverse effects.

At 15 months after surgery, the patient presented with bloody sputum. Chest CT showed multiple endotracheal nodules (Fig. 1C). Sputum cytology revealed squamous cell carcinoma. Bronchoscopy showed multiple protruded endotracheal tumors (Fig. 1D), and a pathological examination of the tracheal tumors revealed squamous cell carcinoma, which was the same histology as the primary lung cancer

${ }^{1}$ Department of Thoracic Surgery, Yamagata Prefectural Central Hospital, Japan and ${ }^{2}$ Department of Pathology, Yamagata Prefectural Central Hospital, Japan

Received: May 20, 2017; Accepted: July 12, 2017; Advance Publication by J-STAGE: November 20, 2017

Correspondence to Dr. Satoshi Shiono, sshiono@ypch.gr.jp 

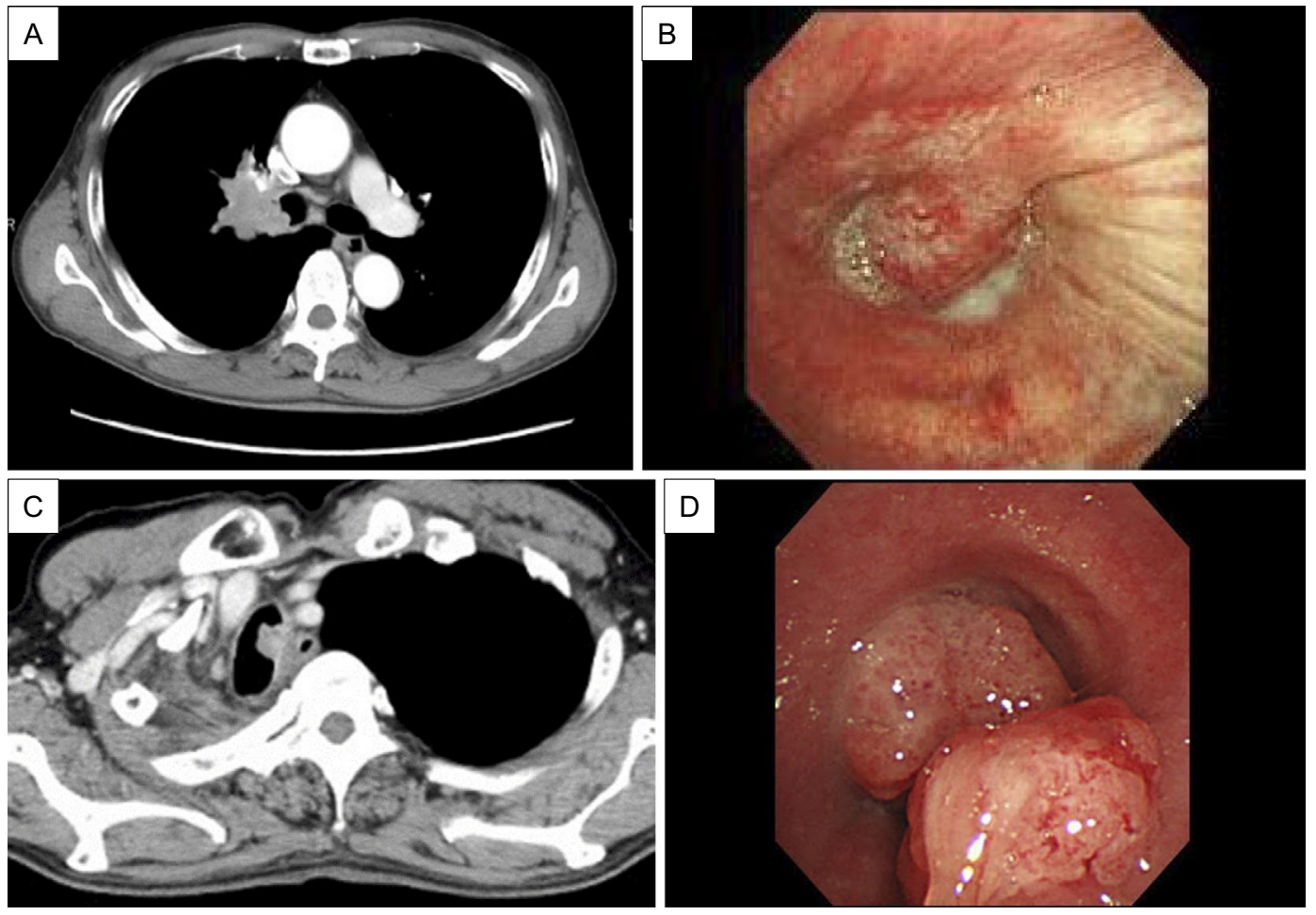

Figure 1. Enhanced computed tomography (CT) of the chest showed a right hilar lung tumor and enlarged right hilar lymph node that invaded the right main bronchus and obstructed the right upper lobe bronchus (A). Bronchoscopy revealed that the tumor completely obstructed the right upper bronchus and invaded the right main bronchus (B). At 15 months after surgery, enhanced CT of the chest showed multiple endotracheal nodules and eccentric tracheal wall thickening (C). Bronchoscopy showed multiple endoluminal protruded tumors in the trachea (D).

(Fig. 2A and B). Furthermore, an immunohistochemical analysis revealed that the resected lung cancer and tracheal tumors were both strongly positive for p53 (Fig. 2C and D). Multiple endotracheal metastases of lung cancer were confirmed. The patient underwent APC therapy using bronchoscopy to avoid the risk of asphyxia and received nanoparticle albumin-bound paclitaxel/carboplatin chemotherapy. He is currently alive at 3 years after the first bronchoscopic treatment and continues to receive chemotherapy.

\section{Discussion}

The incidence of endotracheal metastases from primary lung cancer has been reported to be $0.44 \%$, while that from non-pulmonary malignancies is higher, ranging from $2 \%$ to $50 \%$ (1). Although the most common symptom is hemoptysis, asymptomatic cases have been reported (1). On chest $\mathrm{CT}$, endotracheal metastasis appears as small endotracheal nodules, which should be distinguished from mucous plugs (1). Due to the low incidence, nonspecific symptoms, and ambiguous CT findings, it is challenging to make an early radiological diagnosis of tracheal metastasis.

The mechanisms of endotracheal metastasis of lung cancer have not been clarified (1-5). One possibility is the direct implantation of tumor cells due to bronchoscopic intervention. Youn et al. (4) presented a case of tracheal metastasis of lung cancer and mentioned the possibility of tumor cell implantation due to an endoscopic examination and biopsy. Hu et al. (6) described the possibility of direct implantation in a case of nasopharyngeal metastasis from squamous cell carcinoma of the lung following bronchoscopic therapy. Some cases of implantation metastasis along the biopsy tract after lung tumor needle biopsy have been reported $(7,8)$. Since the present patient underwent bronchoscopic therapy using APC and forceps when he first presented to our hospital suffering from obstructive pneumoniae, a similar process may have occurred. Another possibility is hematogenous and/or lymphatic metastasis. Some reports support this hypothesis based on clinicopathological findings; namely, tumor cells were found in the submucosal lymphatic vessels of the trachea $(1,9)$. In the present case, although hilar lymph node metastasis was confirmed during a histopathological examination following surgery, vascular invasion and lymphatic permeation were not observed. We therefore believe that bronchoscopic therapy might have caused endobronchial metastasis following lung cancer surgery. However, since this was an advanced lung cancer case, it is possible that the lymphatic drainage from hilar lymph node metastasis resulted in endobronchial metastasis. In patients with advanced lung cancer, latent endobronchial metastasis might develop without bronchoscopic therapy.

Bronchoscopic therapy is required for the treatment of patients with central airway obstruction. Various bronchoscopic techniques are available for tracheobronchial tumors, includ- 

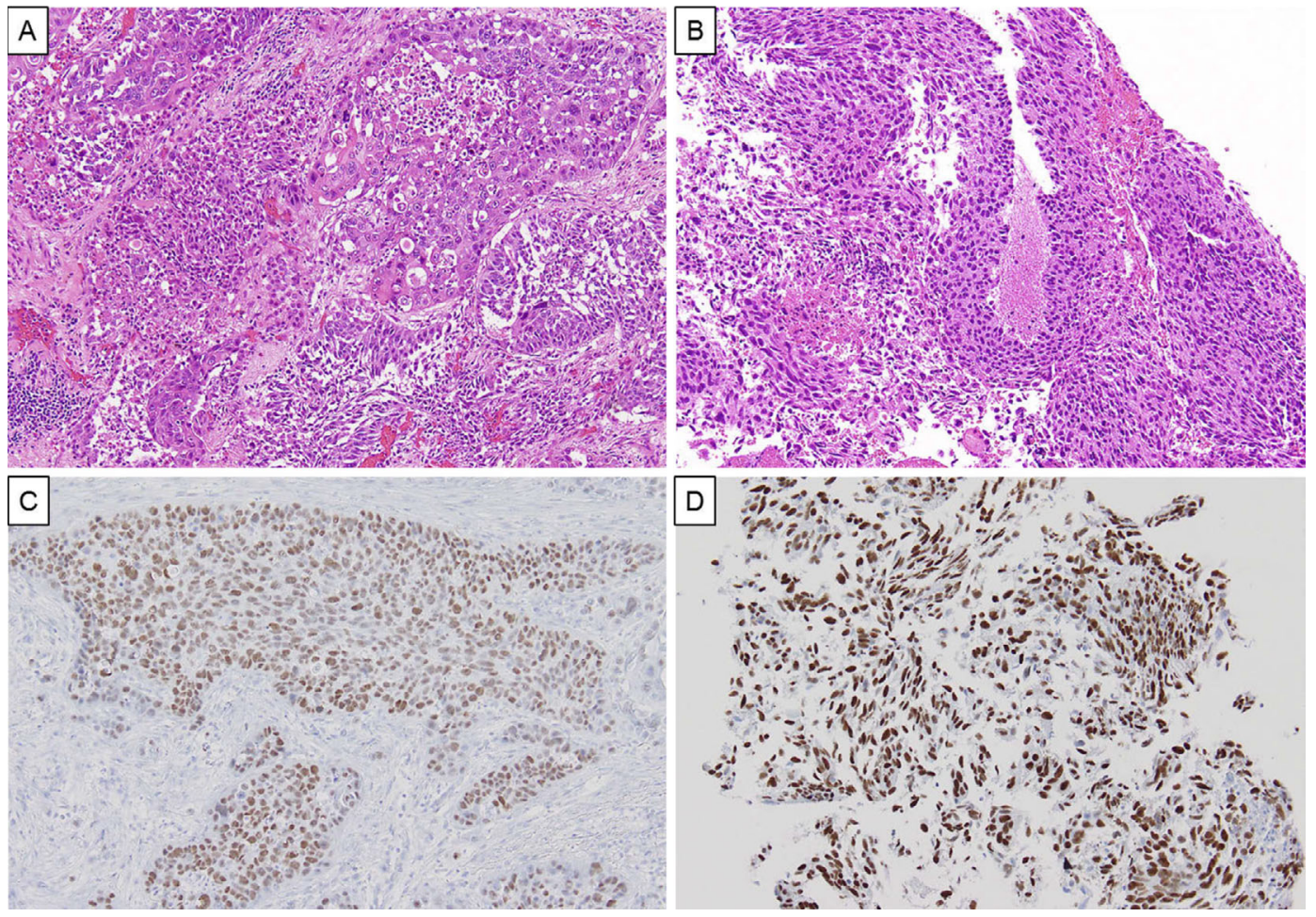

Figure 2. A pathological examination revealed squamous cell carcinoma in specimens of both the primary lung cancer (A) and the tracheal tumors (B) Hematoxylin and Eosin staining. An immunohistochemical analysis revealed that the resected lung cancer $(C)$ and tracheal tumors $(D)$ were both strongly positive for $\mathbf{p 5 3}$.

ing neodymium-yttrium-aluminum-garnet (Nd-YAG) laser therapy, electrocautery, brachytherapy, photodynamic therapy, cryotherapy, and APC. We generally choose APC due to its safety and efficacy; in comparison to Nd-YAG laser therapy, APC has been reported to be less likely to cause serious complications, such as perforation and fatal hemorrhage (10).

In this report, we described an unusual case of multiple endotracheal metastases from lung cancer after bronchoscopic therapy followed by surgery. This report raises the possibility that bronchoscopic therapy may cause the implantation of tumor cells in the airway of lung cancer patients. Bronchoscopic therapy is effective for the treatment of central airway obstruction; however, a careful follow-up is required after it is performed.

The authors state that they have no Conflict of Interest (COI).

\section{References}

1. Chong S, Kim TS, Han J. Tracheal metastasis of lung cancer: CT findings in six patients. AJR Am J Roentgenol 186: 220-224, 2006.

2. Yokoba M, Nishii Y, Hagiri S, Tanimura S, Honma K. Endobronchial metastasis from slow-growing lung cancer: a rare case report and review of the literature. Respir Med CME 1: 107-110, 2008.

3. Kim EY, Kim TS, Choi JY, Han J, Kim H. Multiple tracheal me- tastases of lung cancer: CT and integrated PET/CT findings. Clin Radiol 65: 493-495, 2010.

4. Youn HC, Kim YH, Lee YK, Kim GY. Multiple endotracheal and endobronchial metastases after pneumonectomy for a primary lung cancer: a case report. Thoracic Cancer 4: 453-456, 2013.

5. Zhang Z, Mao Y, Chen H, et al. Endotracheal and endobronchial metastases in a patient with state I lung adenocarcinoma. Ann Thorac Surg 97: 135-137, 2014.

6. Hu JB, Jin M, Chen EG, Sun XN. Lung squamous cell carcinoma metastasizing to the nasopharynx following bronchoscopy intervention therapies: a case report. World J Surg Oncol 12: 68, 2014.

7. Kim JH, Kim YT, Lim HK, Kim YH, Sung SW. Management for chest wall implantation of non-small cell lung cancer after fineneedle aspiration biopsy. Eur J Cardiothorac Surg 23: 828-832, 2003.

8. Kim YD, Lee BY, Min KO, Kim CK, Moon SW. Intrapulmonary recurrence after computed tomography-guided percutaneous needle biopsy of stage I lung cancer. J Thorac Dis 6: 1004-1006, 2014.

9. Kiryu T, Hoshi H, Matsui E, et al. Endotracheal/endobronchial metastases: clinicopathologic study with special reference to developmental modes. Chest 119: 768-775, 2001.

10. Okada S, Yamauchi H, Ishimori S, Satoh S, Sugawara H, Tanabe Y. Endoscopic surgery with a flexible bronchoscope and argon plasma coagulation for tracheobronchial tumors. J Thorac Cardiovasc Surg 121: 180-182, 2001.

The Internal Medicine is an Open Access article distributed under the Creative Commons Attribution-NonCommercial-NoDerivatives 4.0 International License. To view the details of this license, please visit (https://creativecommons.org/licenses/ by-nc-nd/4.0/).

(C) 2018 The Japanese Society of Internal Medicine Intern Med 57: 845-847, 2018 